\title{
Effects of an In-Vehicle Collision Avoidance Warning System on Short- and Long-Term Driving Performance
}

\author{
Avner Ben-Yaacov, Masha Maltz, and David Shinar, Ben-Gurion University of the \\ Negev, Beer-Sheva, Israel
}

\begin{abstract}
Many new in-vehicle systems focus on accident prevention by facilitating the driving task. One such driving aid is an in-vehicle collision avoidance warning system (IVCAWS), used to alert the driver to an impending collision. Our study evaluated the effects of an imperfect IVCAWS both on driver headway maintenance and on driver behavior in response to warning system errors. Our results showed that drivers tend to overestimate their headway and consequently drive with short and potentially dangerous headways, and that IVCAWSs are a useful tool for educating drivers to estimate headway more accurately. Moreover, our study showed that after a relatively short exposure to the system, drivers were able to maintain longer and safer headways for at least six months. The practical implications of these results are that the use of an IVCAWS should be considered for inclusion in driver education and training programs.
\end{abstract}

\section{INTRODUCTION}

In the United States, rear-end collisions represent approximately $30 \%$ of all car crashes on public roads (National Highway Traffic Safety Administration, 1999). Two major causes of such accidents are driver inattention and failure to maintain the proper distance from the lead car (Dingus, Jahns, Horowitz, \& Knipling, 1998). Therefore, a device that can both alert an inattentive driver to an impending crash and help the driver maintain an appropriate distance from the lead car could be an important safety tool.

Two measures are commonly used for converting the distance between vehicles traveling in the same direction into a unit of time. One is time to collision (TTC), or the time it will take for two cars at their present speeds to collide. The second measure, the one used in this study, is temporal headway (TH), the time it will take for the following car to reach the position of the lead car. Although drivers are taught to maintain a safe headway of $2 \mathrm{~s}$ or more from the lead car, and drivers' handbooks provide information about driver reaction time and stopping distance (e.g., Maryland Drivers' Handbook, 1998; National Safety Council, 1992), in real driving situations headways of $1 \mathrm{~s}$ or less are typical of fast rush hour traffic (e.g., Chen, 1996; Evans \& Wasielewski, 1983).

Consequently, past research carried out by Taieb and Shinar (2001) showed that drivers tend to overestimate their headways despite years of driving experience. Evans (1991) suggested three reasons for this risky behavior. First, on the highway, drivers rely on the fact that sudden deceleration by the lead car rarely occurs. Second, they view the lead vehicle speed as a constant, so that if they match its speed, an accident will not occur. Finally, past experience has reinforced such short headway. One reason that drivers tend to misjudge other vehicles' speeds may be related to the difficulty in perceiving external objects' movement in relation to one's own movement (Rumar, 1990).

There have been numerous instances of drivers reporting that they simply did not see the other vehicle until it was too late, commonly referred to as "looking but not seeing" (Storie, 
1977; Treat et al., 1977), the cause of which is probably error in perceptual or cognitive recognition (Crundall \& Underwood, 1997; Rumar, 1990; Storie, 1977).

Driver errors in headway judgment and in the detection of other vehicles' movement lead to the possibility of using technological devices both as a way to educate the driver and as a means to alert the driver to situations that he or she may not have perceived. Such a device measures the $\mathrm{TH}$ and sounds a warning beep when the headway to the lead car is shorter than a predefined threshold.

There are two human factors issues in the implementation of an in-vehicle collision avoidance warning system (IVCAWS). The first is the interface to use in relaying the information from the automatic system to the driver. Some studies compared different methods of warning (visual, auditory, and combinations of the two) and found that most were effective to some degree (Dingus et al., 1997), with an auditory tone being the most effective interface (Hirst \& Graham, 1997; Maltz, Aminov, Aharonov, \& Shinar, 1999).

The second issue is how to analyze the interaction between the driver and the automated warning system when both are capable of error. Sorkin and Woods (1985) recommended that analysis of human performance with an automated aid should be considered a combination of the performance of the automatic system and of the human's subsequent behavior. The automatic system's performance is defined by its probability of detection (of an unsafe headway) and by its probability of a false alarm. The human's behavior is based both on his or her own processing of the event and on the information provided by the automatic system. Some researchers found that human operators will ignore or even disable extremely faulty automatic aids (e.g., Horowitz \& Dingus, 1992; Seminara, Gonzalez, \& Parsons, 1977; Sorkin, 1988), although users can be influenced by the faulty systems even if they mostly ignore them (Maltz \& Meyer, 2001).

In previous research, Taieb and Shinar (2001) found that drivers instructed to drive at a comfortable distance behind a lead car chose a TH of approximately $1 \mathrm{~s}$, irrespective of speed. In the present study, drivers were instructed to maintain a $1 \mathrm{~s} \mathrm{TH}$ to determine whether they were aware of the TH actually maintained. We examined driver response to a headway detection system's warning tone that sounded whenever the driver's headway decreased to less than $1 \mathrm{~s}$ from the lead car. The warning system was programmed to randomly malfunction by generating false alarms (i.e., sounding a tone when TH was longer than $1 \mathrm{~s}$ ) and by missing true events (i.e., not sounding the tone when TH was shorter than $1 \mathrm{~s}$ ).

The following hypotheses are addressed in this paper: (a) Drivers have a poor sense of safe $\mathrm{TH}$ and tend to drive too closely behind the lead car. (b) An IVCAWS will assist a driver in maintaining the proper TH. (c) Use of an IVCAWS can teach a driver to maintain good TH. This learning process will remain long after he or she no longer uses the automatic system. (d) The more reliable the IVCAWS, the better the drivers' performance will be with the system.

\section{METHOD}

Participants. Thirty participants ( 15 women and 15 men) ranging in age from 25 to 50 years took part in the experiment. All were licensed drivers with 5 or more years of driving experience (mean driving experience was 10 years). The participants were evenly divided into three experimental groups. The grouping was based on the reliability level of the warning system.

Equipment. The participants drove a compact car with automatic transmission (1997 Hyundai Accent) and a laser-based headway detection device (ControlLaser model CL200, by Silicon Heights, Ltd.) The ControLaser measures the distance to the lead vehicle by means of a laser beam and an electro-optical system that transmits and processes the data and sounds an auditory signal whenever a predetermined headway has been breached. The device's headway measurement was accurate to within $+/-1 \mathrm{~m}$ in visible conditions (according to the manufacturer's claims). The system provides data, which we collected with a Pentium-grade laptop (166 MHz) at a rate of about $10 \mathrm{~Hz}$, including self- and lead-car speed, distance to the lead car, and TH. The computer sounded the alarm whenever the device detected $\mathrm{TH}<1.0 \mathrm{~s}$; the alarm persisted as long as the headway 
remianed below $1 \mathrm{~s}$. The computer also generated false alarms, which were triggered by the experimenter seated in the passenger seat. In addition, the experimenter muted the alarm at random periods (commensurate with the reliability level of the system for that experimental group) to generate missed alarms.

Procedure. The experiment took place on a six-lane divided highway in the late afternoon under clear skies. Prior to the experiment, participants drove without instruction for about 10 min to the starting point to help familiarize them with the vehicle. Then they were instructed to reach the destination point in minimum time, given the following conditions: (1) Stay close behind some vehicle as much as possible, keeping a $1 \mathrm{~s}$ distance to the lead car, (2) stay in the right lane without overtaking unless instructed to do so, and (3) stay within the speed limit.

The experiment was divided into four trials. In the first trial, participants drove $20 \mathrm{~km}$ (about $15 \mathrm{~min}$ ) with the warning system muted. Before the second trial, they were made aware of the headway detection device and its use as a warning system. For this portion of the experiment, participants were randomly assigned to the three experimental groups, which had different warning system reliability levels. During the 50-minute trial, the warning system was set to malfunction 5,10 , or 20 times to achieve system reliabilities of $95 \%, 80 \%$, and $60 \%$, respectively. The "malfunctions" occurred at random intervals and lasted $30 \mathrm{~s}$. Depending on the headway at the time, either a false alarm was generated by the system (if the headway was long) or the alarm signal was muted (system "miss" if the headway was short). The participants were informed of the system reliability. The second trial was a $70 \mathrm{~km}$ drive (about 50 min) consisting of $35 \mathrm{~km}$ in each direction along the same route. The third trial was the 20 $\mathrm{km}$ drive back to the starting point, with the warning signal again muted.

The fourth trial of the experiment took place 6 months after the participants' initial exposure to the ControlLaser. They drove for $20 \mathrm{~km}$ in the same vehicle as before, along the same road, under the same conditions, and with the same instructions. The warning system was muted for the fourth trial.

\section{RESULTS AND DISCUSSION}

The state of the driver-car warning system was recorded every $300 \mathrm{~ms}$. Four parameters temporal headway to the lead vehicle, driver speed, driver response, and the state of the warning system (beep or no beep) - were grouped into categories. We divided TH into six categories: $0.0-0.4,0.4-0.8,0.8-1.2,1.2-1.6$, 1.6-2.0, and $>2 \mathrm{~s}$ TH. We excluded the last $\mathrm{TH}$ category from all but analyses involving false alarms because it included long stretches with no lead car. Because participants were asked to maintain a $1 \mathrm{~s} \mathrm{TH}$, the first two categories were classified as within the danger zone, the last two categories as out of the danger zone, and the 0.8-1.2 s TH category as the requested driving performance (allowing for noise in either direction). The warning system's state was either sounding an alert (beep) or not (no beep). The potential driver responses were to slow down (noted whenever vehicle speed decreased by at least $3 \%$ for at least $1.5 \mathrm{~s}$ ), to speed up (in which speed similarly increased), or to maintain current speed. If a change in speed occurred more than two seconds after the alarm sounded, it was not considered a response to the alarm. Driver speed was assigned to two categories: fast $(>90 \mathrm{~km} / \mathrm{h})$ or slow $(\geq 90$ $\mathrm{km} / \mathrm{h}$ ).

Figure 1 presents the percentage of time spent by the participants in each TH category on each of the trials. A two-way analysis of variance (ANOVA) was computed (trial [4] $\times$ $\mathrm{TH}$ [5]) on percentage of time in the TH category as the dependent variable. The two-way interaction was significant, $F(12,348)=24.86$, $p<.0001$. Individual ANOVA tests comparing $\mathrm{TH}$ in the first trial with the three trials during, immediately after, and six months after exposure to the IVCAWS all yielded significant differences, $F(4,116)=43.67, p<.0001, F(4$, $116)=34.86 ; p<.0001$, and $F(4,116)=24.9$, $p<.0001$, respectively.

In the first trial, the drivers spent an average of $42.2 \%$ of the time in the danger zone (TH $</=0.8 \mathrm{~s}$ ). During and after the use of the ControLaser, this percentage dropped to $3.5 \%$ and $6.5 \%$, respectively, compared with $22.8 \%$ before exposure to the device. The key result was that there were no statistical differences 


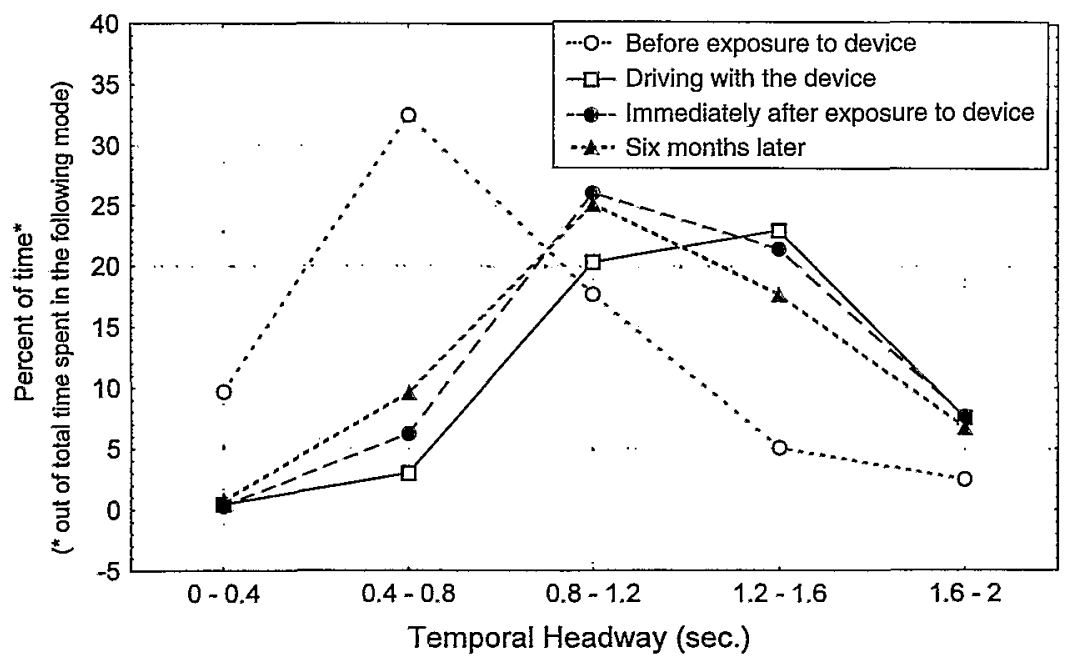

Figure 1. Drivers' headways in the four experimental trials.

between the third trial (immediately after exposure to the device) and the fourth trial ( 6 months later), $F(4,116)=1.4, p=0.25$, yet there was a statistical difference between preexposure headways and the headways in the fourth trial (see above), showing a long-term learning effect of headway maintenance. In the fourth trial, TH was less than $0.8 \mathrm{~s}$ for an average of $10.4 \%$ of the time, and between $0.8 \mathrm{~s}$ and $1.6 \mathrm{~s}$ for $42.8 \%$ of the time.

To test whether the system's reliability level influenced the headway maintenance, we measured the percentages of time spent in the different TH categories as a function of the reliability of the system both during exposure to the device and in the trial immediately following exposure to the device $-\mathrm{TH}$ (5) $\times$ Reliability (3). No significant effects of system reliability were found in driver performance in either of the trials, $F(8$, $108)=1.07, p=.39$, and $F(8,108)=0.78, p=$ .63 , respectively. In the second trial, during exposure to the IVCAWS, the participant groups with the high, medium, and low reliability levels had headways between 0.8 for an average of $2.7 \%, 4.6 \%$. And $3.1 \%$ of the time, respectively, and headways between $0.8 \mathrm{~s}$ and $1.6 \mathrm{~s}$ for $42.5 \%, 40.6 \%$, and $47 \%$ of the time, respectively. In the trial immediately following exposure to the IVCAWS, the groups averaged $3.8 \%$, $10.4 \%$, and $5.5 \%$ of the time, respectively, with $\mathrm{TH}<0.8 \mathrm{~s}$, and $48.7 \%, 45.7 \%$, and $48.1 \%$ of the time, respectively, and with headways between 0.8 and $1.6 \mathrm{~s}$.

We included the TH category of $>2 \mathrm{~s}$ when studying the effects of driver speed on driver behavior during and after exposure to the ControlLaser. This was done because we assumed that an open roadway gave the drivers an opportunity to increase their speed, and we wished to see if drivers who tended to exceed the speed limit would exhibit headways that were different when receiving alerts than when not receiving alerts. A three-way ANOVA - Driver Speed (2) $\times \mathrm{TH}(6) \times$ Trial (2) - with trial treated as a repeated measure, was run on the percentage of time in each $\mathrm{TH}$ condition. There were no effects of driver speed on headway maintenance, $F(5,145)=0.79, p=.56$, indicating that the effects of the IVCAWS were consistent across driving speed categories. In the second trial, while driving with the IVCAWS, the slower drivers averaged $4 \%$ of the time in $\mathrm{TH}<0.8 \mathrm{~s}$ and $49 \%$ in TH between 0.8 and 1.6 s compared with $3.3 \%$ and $39.9 \%$, respectively, for the faster drivers. In the third trial, following exposure to the IVCAWS, the slower drivers averaged $6.5 \%$ of the time in $\mathrm{TH}<0.8 \mathrm{~s}$ and $50.4 \%$ in $\mathrm{TH}$ between 0.8 and $1.6 \mathrm{~s}$, and the faster drivers averaged $6.9 \%$ and $43.8 \%$, respectively.

We defined four IVCAWS conditions of interaction between the independent variables 
$\mathrm{TH}$ and warning state: (a) true sounding of the alert $\mathrm{TH}$ in the danger zone and warning state of beep), (b) false-alarm alert (TH outside the danger zone and warning state of beep), (c) warning system miss of an event ( $\mathrm{TH}$ in the danger zone and warning state of no beep), and (d) proper nonalert (TH outside of the danger zone and warning state of no beep). We measured the relative frequencies of these conditions for all categories of $\mathrm{TH}$ except $\mathrm{TH}=0.8$ to $1.2 \mathrm{~s}$ because this $\mathrm{TH}$ included the desired headway and could not be classified as totally within or totally outside of the danger zone.

Figure 2 shows driver behavior (excluding the response of no speed change) in response to the warning system's alert or nonalert when in the danger zone. A three-way ANOVA was computed on the two short TH categories: Warning State $(2) \times \mathrm{TH}(2) \times$ Driver Response (2). A significant interaction between warning system state and response, $F(1,29)=17.98, p<$ .0002 , showed that drivers rarely speeded up in response to the warning and were more likely to decrease their speed when the system alerted them to their short headways than when the system did not alert them. Under conditions of true warning (i.e., $\mathrm{TH}<0.8 \mathrm{~s}$ and warning beep on), the drivers slowed in response to the beep an average of approximately $45 \%$ of the time and accelerated an average of $5 \%$ of the time (for the remaining time, speed remained constant).
This contrasted with the cases in which there were no warning beeps and the drivers were within the danger zone. Here the drivers slowed an average of only $23 \%$ of the time. Accelerating remained the same as for true alerts. Thus the alarm doubled the rate of correct responses to short THs.

To examine driver response to false alarms, a three-way ANOVA was run with the three long. TH categories: Warning State $(2) \times \mathrm{TH}$ (3) $\times$ Driver Response (2). There was a significant interaction between warning system state and response, $F(1,29)=6.14, p<.02$. As can be seen in Figure 3, false alerts did not particularly cause unnecessary speed reductions. Speed reductions remained at about $10 \%$, regardless of the activation (false alarm) or nonactivation (correct nonalerting) of the IVCAWS. However, false alerts affected the drivers' tendency to speed up. Without alerts, drivers sped up about $20 \%$ of the time when they were outside the danger zone, but with the false-alarm alerts they sped up only $11 \%$ of the time.

An examination of the 0.8-1.2 $\mathrm{s}$ TH category - a "borderline" condition that encompassed the typical headway and the one that the participants were instructed to maintain - yielded a significant difference in driver behavior before and after exposure to the warning system. A two-way ANOVA - Driver Slow Down or Speed Up (2) $\times$ Trial (4) - was computed on

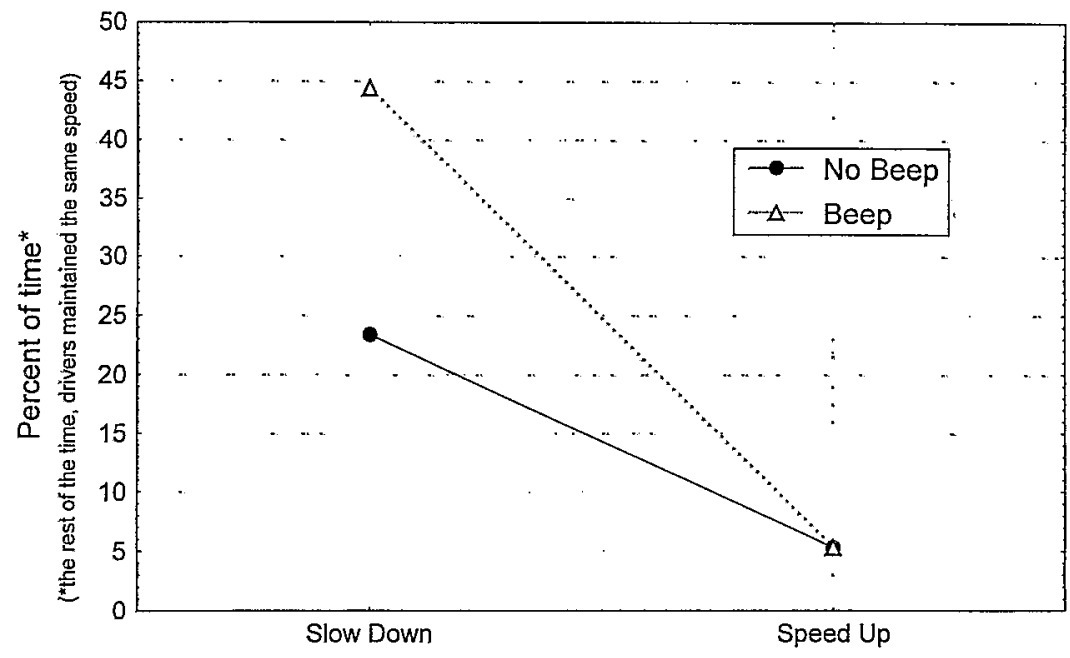

Figure 2. Percentage of responses to beeps/misses in the danger zone ( $\mathrm{TH} \geq 0.8 \mathrm{~s}$ ). 


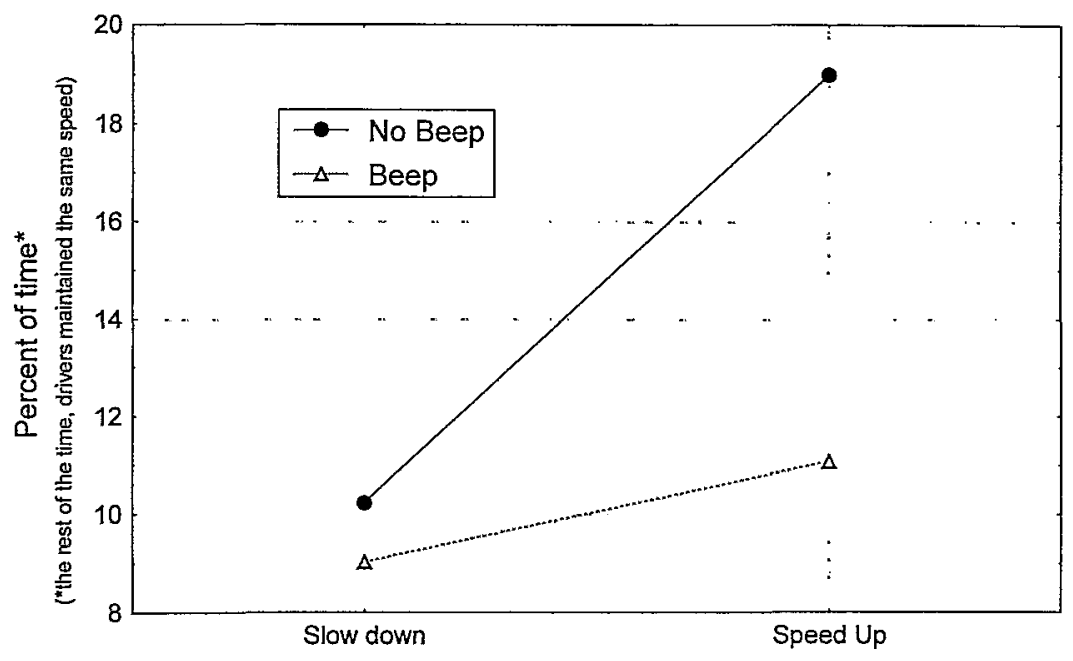

Figure 3. Percentage of responses to false alarms/correct nonalerting in the safe zone (TH > $1.2 \mathrm{~s})$.

the time spent in the borderline headway zone, yielding a significant interaction between driver behavior and trial, $F(3,87)=22.07, p<.0001$. As illustrated in Figure 4, whereas in the trial before exposure to the warning system, the percentages of time that the drivers slowed down and accelerated while in the borderline zone were approximately equivalent (around 14\%), during all other trials drivers slowed down more often than they sped up, showing a heightened sensitivity to the headway. Note that in this $\mathrm{TH}$ category the alarm was sounded whenever $\mathrm{TH}$ was $<1.0 \mathrm{~s}$, so that as far as the drivers were concerned, they were getting a true alert.

\section{SUMMARY AND CONCLUSIONS}

Most of our hypotheses, stated earlier, were confirmed by our results. First, we found that drivers are generally poor at estimating temporal headway. Prior studies reported driver error in estimation of TH at between $20 \%$ and $42 \%$

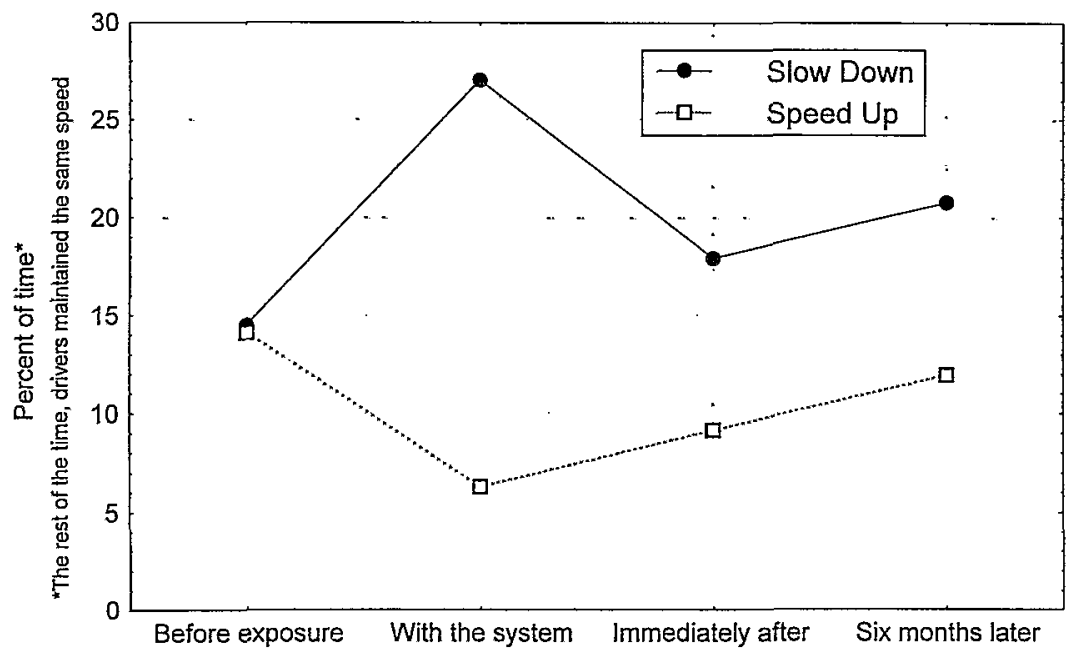

Figure 4. Driver behavior in the "borderline" zone ( $\mathrm{TH}=0.8-1.2 \mathrm{~s})$. 
(Cavallo, Laya, \& Laurent, 1986; Hoffman \& Mortimer, 1994; McLeod \& Ross, 1983). The reports, based on laboratory studies, were confirmed in our study and in the study by Taieb and Shinar (2001), which were performed under actual road conditions. Drivers' headway estimation, however, can be improved with an IVCAWS such as the one we employed. During use of the headway detection device, the drivers' headway increased dramatically.

In addition, use of the IVCAWS taught the drivers to correctly assess TH, and they were able to maintain safer headways, both immediately after being exposed to the system and after six months - a significant time, considering the lack of feedback during the period. Although we cannot verify whether or not our participants actually changed their habitual THs after their exposure to the headway detection system, in the postdriving debriefing they all commented on changes in their habits with statements such as, "I keep much longer headways now" and "Now I know what headway I should be maintaining." Thus the delayed fourth trial demonstrated that participants' newfound ability to estimate and maintain $\mathrm{TH}$ was firmly established.

It is reassuring to note that the warning system did not have to be perfect to be useful. Dingus et al. (1997) showed that a warning system of less than $60 \%$ accuracy was not effective. Our results (contrary to our hypothesis) showed that there were no significant differences among $60 \%, 80 \%$, and $95 \%$ reliable systems. The drivers were somewhat affected by the false alarms, slowing down unnecessarily, and occasionally they did not slow down when $\mathrm{TH}$ was in the danger zone and the alert was not sounded. However, overall, the headways maintained by the drivers were not significantly different with the different levels of IVCAWS reliability. Apparently the combination of the warning system and the natural perception of the driver concerning where he or she was located in relation to the lead vehicle provided enough input for the driver to process headway maintenance information optimally. These results were independent of driver speed.

Because maintaining adequately safe headway is universally defined as a desired characteristic of safe driving, it appears that a training period with a warning system such as the one we used in the study, followed by constant and significant encouragement to maintain a safe temporal headway, should lead to safer driver behavior.

It is important to keep in mind that IVCAWS can be effective in improving headways when drivers are motivated, as in our study. Our results do not imply that, given feedback, drivers will choose of their own free will to adjust their headways accordingly. This is a basic difference between optimal performance (as measured here) and habitual behavior (as reflected in reallife driving). Further research is now being conducted to see the effects of IVCAWS on habitual driving behavior.

One direct implication of the effects of IVCAWS on driving behavior is that people can benefit from valid feedback and can learn quickly to keep safe headways. One way to achieve that for people who don't have such a device is by installing a sensor in the rear of the car to alert following drivers when they approach too closely. This would also provide a means of communication between drivers. A study to evaluate this mode of operation is underway.

Another potential use for IVCAWS that warrants study is the integration of IVCAWS into intelligent cruise control. The IVCAWS would signal the system whenever the chosen speed compromises safe headway to a slower lead car, and the cruise control would automatically disengage the accelerator.

\section{ACKNOWLEDGMENTS}

We thank Yael Edan for providing us with some of the software for the data retrieval. Part of this study was presented at the International Ergonomics Association XIVth Triennial Congress/Human Factors and Ergonomics Society 44th Annual Meeting.

\section{REFERENCES}

Cavallo, V., Laya, O., \& Laurent, M. (1986). The estimation of time to collision as a function of visual stimulation. In A. G. Gale, M. H. Freeman, C. M. Haslegrave, P. Smith, \& S. P. Taylor (Eds.), Proceedings of vision in vehicles (pp. 179-183). Amsterdam: Elsevier.

Chen, S. K.(1996). Estimation of car-following safety: Application to the design of intelligent cruise control. Dissertation, Massachusetts Institute of Technology, Cambridge, MA. 
Crundall, D., \& Underwood, G. (1997). Concurrent verbalisation during drivers' visual search and hazard perception. In T. Rthergatter \& E. Carbonell-Vaya (Eds.), Traffic and transportation psychology: Theory and application (pp. 121-135). Amsterdam: North Holland.

Dingus, T. A., Jahns, S. K., Horowitz, A. D., \& Knipling, R. (1998). Human factors design issues for crash avoidance systems. In W. Barfield \& T. A. Dingus (eds.), Human factors in intelligent transportation systems (pp 55-94). Mahwah, NJ: Erlbaum.

Dingus, T. A., McGehee, D. V., Mankkal, N., Jahns, S. K, Camey, C. \& Hankey, J. M. (1997). Human factors field evaluation of automotive headway maintenance/collision warning devices. Human Factors, 39, 216-229.

Evans, L. (1991). Traffic safety and the driver. New York: Van Nostrand Reinhold.

Evans, L., \& Wasielewski, P. (1983). Risky driving related to driver and vehicle characteristics. Accident Analysis and Prevention, $15,121-136$.

Hirst, S., \& Graham, R. (1997). The format and presentation of collision warnings. In Y. I. Noy (Ed.), Ergonomics and safety of intelligent driver interfaces (pp. 203-219). Mahwah, NJ: Erlbaum.

Hoffmann, E. R., \& Mortimer, R. G. (1994). Driver's estimates of time-to-collision. Accident Analysis and Prevention, 26, $511-520$.

Horowitz, A. D., \& Dingus, T. A. (1992). Warning signal design: A key human factors issue in an in-vehicle front-to-rear-end collision warning system. In Proceedings of the Human Factors Society 36th Annual Meeting (pp. 1011-1013). Santa Monica, CA: Human Factors and Ergonomics Society.

Maltz, M., Aminov, I., Aharonov, S., \& Shinar, D. (1999). Driver responses to imperfect collision warning cues. In Proceedings of vision in vehicles VIII. Amsterdam: North Holland.

Maltz, M., \& Meyer, J. (2001). Use of warnings in an attentionally demanding detection task. Human Factors, 43, 217-226.

Maryland Driver's Handbook. (1998). Glen Burnie, MD: Maryland Department of Transportation.

McLeod, R. W., \& Ross, H. E. (1983). Optic flow and cognitive factors in time to collision estimates. Perception, 12, 417-423.

National Safety Council. (1992). Defensive driving course [Course guide]. Itasca, IL: Author:

National Highway Traffic Safety Administration. (1999). Traffic safety facts 1998: A compilation of motor vehicle crash data from the Fatality Analysis Reporting System and the General Estimates System (DOT HS 808 983). Washington, DC: U.S. Department of Transportation.

Rumar. K. (1990). The basic driver error: Late detection. Ergonomics, 33, 1281-1290.
Seminara, I. L., Gonzalez, W. R., \& Parsons, S. O. (1977). Human factor review of nuclear power plant control room design (NP. 309). Palo Alto, CA: Electric Power Research Institute.

Sorkin, R. D. (1988). Why are people turning off our alarms? Journal of the Acoustical Society of America, 84, 1107-1108.

Sorkin, R. D., \& Woods, D. D. (1985). Systems with human monitors: A signal detection analysis. Human Factors and Ergonomics: Computer Interaction, 1, 49-75.

Storie, V. I. (1977). Male and female car drivers: Differences observed in accidents (TRRL Report LR761). Crowthorne: Transport Research Laboratory.

Taieb, M., \& Shinar, D. (2001). Minimum and comfortable driving headways: Reality vs. perception. Human Factors, 43, 159-172.

Treat, J. R., Tumbas, N. S., McDonald, S. T., Shinar, D., Hume, R. D., Mayer, R. E.. Stansifer, R. L.. \& Castellan, N. J. (1977, March). Tri-level study of the causes of traffic accidents (Indiana University Final Report. U.S. Department of Transportation Report No. DOT-HS-034-3-535-77-TAC). Washington, DC: U.S. Department of Transportation.

Avner Ben-Yaacov is a Ph.D. candidate in human factors engineering at the Department of Industrial Engineering and Management, Ben-Gurion University of the Negev. He received his MBA in human factors engineering from the same university, and this study is part of his graduate thesis.

Masha Maltz is a research scientist at the Massachusetts Institute of Technology's AgeLab. She received her Ph.D. in human factors engineering from the Department of Industrial Engineering and Management, Ben-Gurion University of the Negev, in 2001.

David Shinar is a professor of ergonomics at the Department of Industrial Engineering and Management, Ben-Gurion University of the Negev. He received his $\mathrm{Ph} . \mathrm{D}$. in human performance and human factors engineering from Ohio State University in 1973.

Date received: February 22, 2001

Date accepted: August 10, 2001 


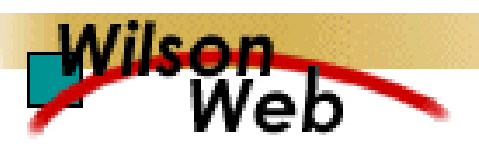

\section{COPYRIGHT INFORMATION}

TITLE: Effects of an In-Vehicle Collision Avoidance Warning System on Short- and Long-Term Driving Performance

SOURCE: Hum Factors 44 no2 Summ 2002

WN: 0219602939012

The magazine publisher is the copyright holder of this article and it is reproduced with permission. Further reproduction of this article in violation of the copyright is prohibited.

Copyright 1982-2002 The H.W. Wilson Company. All rights reserved. 